\title{
Philosophiques
}

\section{Droit naturel et droit positif chez saint Thomas d'Aquin}

\section{Léon Charette}

Volume 8, numéro 1, avril 1981

URI : https://id.erudit.org/iderudit/203152ar

DOI : https://doi.org/10.7202/203152ar

Aller au sommaire du numéro

Éditeur(s)

Société de philosophie du Québec

ISSN

0316-2923 (imprimé)

1492-1391 (numérique)

Découvrir la revue

Citer cet article

Charette, L. (1981). Droit naturel et droit positif chez saint Thomas d'Aquin.

Philosophiques, 8(1), 113-130. https://doi.org/10.7202/203152ar d'utilisation que vous pouvez consulter en ligne.

https://apropos.erudit.org/fr/usagers/politique-dutilisation/ 


\title{
DROIT NATUREL ET DROIT POSITIF CHEZ SAINT THOMAS D'AQUIN
}

\author{
par Léon Charette
}

Le présent article veut examiner les différentes sortes de préceptes rattachés par saint Thomas d'Aquin à la loi naturelle, ainsi que le double processus de dérivation des préceptes admis par lui. En cours de route, il faudra parler, entre autres, des préceptes du Décalogue, du droit des gens, et de questions particulières telles que les modalités d'exercice du droit de propriété, les formes de mariage et les formes de gouvernement. La conclusion consistera à faire voir implicitement qu'en raison même de la complexité inhérente à cette théorie, la vision de la loi naturelle de saint Thomas d'Aquin ne peut en aucune façon être décrite comme une théorie purement déductive, mais qu'au contraire, la part du raisonnement empirique et du mode de détermination y occupe de loin la place prépondérante.

1 - Notions fondamentales. Parmi les notions fondamentales les plus essentielles à l'étude de la question qui nous occupe, il faut mentionner en premier lieu l'existence de la loi éternelle qui, pour saint Thomas, n'est autre que la Divine Sagesse par laquelle «Dieu est également celui qui gouverne tous les actes et tous les mouvements que l'on remarque en chaque créature ${ }^{1}$.» Et partant de là, saint Thomas conclut: «Il est donc évident que la loi naturelle n'est pas autre chose qu'une participation de la loi éternelle dans la créature raisonnable $e^{2}$. Précisons que chez l'être raisonnable, cette participation à la loi éternelle se présente tant sous la forme de la connaissance que sous la forme d'inclinations ${ }^{3}$. Tout cela permet de fonder théologiquement l'adage qui pourrait

1. IalI ae, q. 93 , a.1. Voir aussi De Ver., q. 23 , a.1. C'est pourquoi en précisant quelque peu, il pourra définir la loi comme «une ordonnance de la raison en vue du bien commun, établie et promulguée par celui a charge de la communauté". IaIIae, q. 90, a.4.

2. Q.91, a.2. (En l'absence d'indication contraire, les références à la Somme Théologique renvoient à la $I^{a} I^{a \ell}$.)

3. Q. 91, a.2; q. 93, a.6. 
à la rigueur être suffisamment fondé sur des arguments d'ordre exclusivement philosophique, à savoir Agere sequitur esse.

Dans notre contexte, cela revient à dire que chaque être est appelé à se réaliser selon sa nature propre, et que, dans le cas de l'homme, ceci implique nécessairement le recours à sa propre raison ${ }^{4}$. C'est parce qu'il appartient à la raison humaine d'être règle et mesure des actions humaines ${ }^{5}$, que les premières propositions de l'ordre pratique ne sont pas seulement des principes, mais aussi des préceptes ${ }^{6}$. Ces préceptes jouent donc, dans l'ordre pratique, le même rôle que les premiers principes dans l'ordre spéculatif, et ils sont acquis par un habitus intellectuel qui prend le nom de syndérèse ${ }^{7}$.

Les préceptes de la loi naturelle sont de plus élaborés à partir du concept de bien et sur la base des inclinations fondamentales de la nature humaine. Ainsi, le premier principe fondé sur la notion de bien, "Le bien est ce que toutes choses désirent", se double d'un premier précepte: «Il faut faire le bien et éviter le mal». Mais,

Puisque le bien a la nature d'une fin, et le mal la nature d'un contraire, il s'ensuit que toutes les choses pour lesquelles l'homme a une inclination naturelle sont naturellement appréhendées par la raison en tant que bien et sont par conséquent objets de désir, comme leurs contraires sont mauvais et objets de fuite. C'est pourquoi l'ordre des préceptes de la loi naturelle correspond à l'ordre des inclinations naturelles ${ }^{8}$.

À la limite, mais cette fois sans parler de préceptes, saint Thomas ira même jusqu'à dire: «À chaque inclination naturelle nettement définie correspond une vertu spéciale ${ }^{9} »$. Mais au niveau le plus général d'abord, la raison partira du premier précepte et de l'inclination générale au bien qu'il exprime, pour interpréter les grandes inclinations spécifiques et les traduire en préceptes distincts. Voyons maintenant comment se présente, dans son ensemble, l'articulation des principaux types de préceptes rattachés au droit naturel.

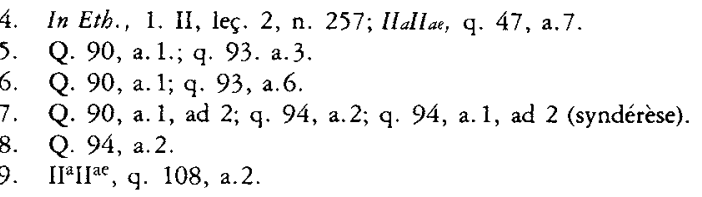


2 - Les trois "degrés» ou types de préceptes. Saint Thomas distingue différents types de préceptes en de nombreux passages de ses textes. Mais deux articles de la question 100 fournissent le cadre le plus englobant pour situer tout ce qu'il dit à ce sujet. Citons notamment l'article 3:

Il y a deux sortes de préceptes qui ne sont pas du nombre des préceptes du Décalogue: d'une part, les préceptes premiers et généraux, qui n'ont pas besoin d'être déclarés autrement que par leur insertion dans la raison naturelle au titre de connaissance immédiate, comme de ne faire de tort à personne et autres du même genre; et, d'autre part, ceux dont la convenance se découvre aux sages après une étude attentive, car c'est à travers l'enseignement des sages que ces préceptes parviennent de Dieu au peuple ${ }^{10}$.

Ces préceptes premiers et généraux, qui sont antérieurs au Décalogue, saint Thomas n'en donne nulle part une énumération complète parce qu'ils sont connus plutôt par mode de connaturalité affective et se rattachent assez nettement aux grandes inclinations de la nature humaine ${ }^{11}$. Il mentionne d'abord expressément: il faut faire le bien et éviter le mal ${ }^{12}$, il ne faut faire de tort à personne ${ }^{13}$, il faut aimer Dieu et son prochain ${ }^{14}$. Pour les autres préceptes du même type (on dit parfois préceptes primaires du droit naturel), saint Thomas ne donne que des indications génériques. Aux inclinations que l'homme partage avec tout être, et qui visent pour toute substance la conservation de son être, il rattache les préceptes relatifs à la conservation de la vie humaine; aux inclinations que l'homme partage en commun avec les autres animaux, il rattache les préceptes se rapportant à l'union des sexes et à l'éducation de la progéniture; aux inclinations d'ordre rationnel que l'homme possède en propre, il rattache le «désir naturel de connaître la vérité sur Dieu et de vivre en société ${ }^{15}{ }_{\text {» }}$.

10. Q. 100, a.3.; a. 11 .

11. Q. 94, a.2. Sur la connaissance par connaturalité affective, voir $I_{a}$ Pass, q. 1, a.5, ad 3; II $^{\mathrm{a}} \mathrm{I}^{\mathrm{ac}}$, q. 45 , a. 2.

12. Q. 94, a. 2. Suppl., q. 65, a. 1, ad 7: «ne fais pas aux autres ce que tu ne voudrais pas que l'on te fasse».

13. Q. 95 , a.5; q. 100 , a.3.

14. Q. 100, a.11; IIallae, q. 44, a. 1, ad 3.

15. Q. 94, a.2. Pour une formulation un peu plus précise de ces préceptes, J. Maritain ( L'Homme et l'État, Paris, P.U.F., 2e éd. 1965, p. 86) suggère: "par exemple: ôter la vie à un homme est chose plus grave qu'ôter la vie à un animal quelconque; ou bien: le groupe familial a à obéir à un type de structure fixe; ou bien: les relations sexuelles ont à être soumises à certaines limitations données; ou bien: nous sommes tenus de nous tourner vers la réalité de l'Invisible; ou bien: nous sommes tenus de vivre ensemble sous certaines règles et prohibitions.» 
Viennent en second lieu les préceptes dits secondaires, ceux du Décalogue ${ }^{16}$. Ce sont, dit saint Thomas, ceux qui se rattachent aux préceptes premiers par mode de conclusion très rapprochée $^{17}$ et pour lesquels une brève réflexion suffit ${ }^{18}$. Ces préceptes ne considèrent pas les actions de l'homme pris individuellement, mais seulement dans la mesure où ces actions peuvent être ordonnées à autrui: Dieu et les autres hommes. C'est pourquoi ils relèvent de la justice et se rapportent au bien commun ${ }^{19}$. Ils n'incluent que ce qui est strictement nécessaire à l'ordre vertueux de la sociétée ${ }^{20}$. Entrent également dans cette catégorie certains préceptes du droit des gens sur lequel nous reviendrons un peu plus loin, par exemple le précepte que les contrats et traités doivent être respectés.

Viennent en dernier lieu les préceptes dits tertiaires qui se rattachent aux précédents à titre de conclusions éloignées; ils exigent une réflexion plus longue et attentive, de même que l'apport des sages ${ }^{21}$, sans cesser pourtant d'appartenir à la loi naturelle ${ }^{22}$. Ce sont de nouvelles déterminations ajoutées aux préceptes du Décalogue ${ }^{23}$, par exemple l'interdiction du culte des dieux étrangers, la prohibition du parjure, les égards dûs aux vieillards, la prohibition de la haine et de l'agression, l'interdiction de la prostitution, de l'usure, des faux jugements et du mensonge. Il faut enfin mentionner à part les préceptes cérémoniels et judiciaires (lois positives) pouvant encore conserver un certain lien avec la loi naturelle. Cependant, les deux derniers types ou degrés de préceptes mentionnés, soit les préceptes secondaires et tertiaires, exigent déjà que l'on fournisse certaines précisions sur la façon dont ils se relient aux préceptes premiers les plus communs.

3 - Le double mode de dérivation des préceptes. Ainsi qu'il y a été fait allusion dans les paragraphes précédents, saint Thomas considère qu'il est possible d'une double manière de dériver des

\footnotetext{
16. Q. 100 , a.1 et a.11.

17. Q. 94 , a.5 et a.6

18. Q. 100, a.3.

19. Q. 100 , a.11, ad 3 .

20. Q. 99 , a. 5 .

21. Q. 100, a.3.

22. Q. 100 , a. 1 .

23. Q. 100 , a. 11 , ad 2 .
} 
préceptes additionnels en partant des principes premiers et communs:

D'une part, comme des conclusions par rapport aux principes; d'autre part, comme des déterminations quelconques de règles générales et indéterminées. Le premier mode ressemble à celui des diverses sciences où les conclusions démonstratives se tirent des principes. Quant au second mode, il ressemble à ce qui se passe dans les arts quand les modèles communs sont déterminés à une oeuvre particulière; tel est le cas de l'architecte qui doit préciser la détermination générale de la forme «maison» à telle ou telle forme d'habitation ${ }^{24}$.

Et dans la suite du même passage, on trouve les deux exemples suivants. C'est à titre de conclusion que du précepte «il ne faut pas faire le mal", on pourra déduire le précepte secondaire «il ne faut pas tuer». Et c'est à titre de détermination que du précepte «celui qui commet une faute doit être puni», on pourra tirer «il sera puni de telle peine particulière». Il est sans doute évident que les deux processus de raisonnement ne sont pas identiques. Dans le premier cas, il s'agit de savoir si le contenu du précepte secondaire entre dans l'extension logique du premier. Ce n'est manifestement pas la question posée dans le second cas. Il s'agit plutôt de savoir s'il y a un rapport de convenance entre telle faute et telle peine, compte tenu des circonstances contingentes de temps et de lieu. Aucune déduction purement logique ne permettra jamais d'établir un lien absolument nécessaire entre les deux termes.

Il peut être important ici de faire déjà remarquer qu'en général, les préceptes d'ordre moral, notamment le Décalogue et ses additions, sont dérivés des principes généraux et communs selon le premier mode, tandis que les préceptes cérémoniels et judiciaires, c'est-à-dire la loi civile, le sont plutôt par mode de détermination, en tenant compte des circonstances sociales changeantes $^{25}$. Passons donc immédiatement à l'étude du Décalogue.

4 - Les préceptes du Décalogue. Rappelons d'abord brièvement pour mémoire que les préceptes premiers et communs de la loi naturelle sont ceux qui nous apparaissent comme évidents par eux-mêmes, notamment en ce qui concerne les trois grands ordres

24. Q. 95, a.2.

25. Q. 95 , a.2; q. 99 , a.4. 
d'inclinations inhérents à la nature humaine ${ }^{26}$. C'est à ce premier niveau qu'appartiennent également les deux grands préceptes de l'amour de Dieu et du prochain «que la raison humaine connaît immédiatement, par la nature ou par la foi. Et ainsi tous les précepts du Décalogue se rapportent à ces deux-là comme les conclusions aux principes généraux ${ }^{27}$." Mais regardons les choses d'un peu plus près.

Il semble bien, d'une part, que les préceptes relatifs au prochain (seconde table du Décalogue) soient plus faciles à saisir que ceux relatifs à Dieu (première table). En effet, lorsque saint Thomas donne des exemples explicites de ces préceptes dont l'élaboration ne requiert qu'une brève réflexion, ces exemples sont tous tirés de la seconde table ${ }^{28}$. D'autre part, saint Thomas avait écrit dans le même passage: "Il y a enfin d'autres préceptes que l'homme ne peut discerner sans l'aide d'une révélation divine, comme il arrive en matière de foi, ( . . p par exemple: Tu ne feras pas d'image taillée ni de représentation, tu ne prendras pas en vain le nom de ton Dieu.» Quelques articles plus loin, il précise:

Appartiennent donc au Décalogue les préceptes dont l'homme reçoit lui-même de Dieu la connaissance. Or c'est le cas de ceux qu'une brève réflexion suffit à faire conclure des préceptes généraux, et aussi de ceux que nous fait immédiatement connaitre la foi infusée par Dieu ${ }^{29}$.

Le grand commandement de l'amour de Dieu et les préceptes relatifs à Dieu (première table) semblent donc impliquer une connaissance de la nature de Dieu qui pourrait bien être au-dessus des capacités naturelles de la raison, et c'est apparemment pour qu'une connaissance adéquate de ces préceptes nous soit accessible qu'une quelconque intervention divine est requise. D'ailleurs, si l'existence même de Dieu n'est pas évidente et ne peut être prouvée qu'avec les difficultés que l'on sait ${ }^{30}$, comment sa nature le serait-elle, de même que les préceptes moraux qui en découlent? On se souviendra ici de l'argumentation par laquelle saint Thomas montre le caractère convenable de la révélation, même en ce qui concerne les connaissances accessibles à la raison naturelle

26. Q. 94, a.2.

27. Q. 100, a.3, ad 1.

28. Q. 100 , a.1.

29. Q. $100, a .3$

30. I'Pars, q. 2 , a. 1-3. 
au sujet de Dieu: très peu d'hommes y parviendraient, mais avec beaucoup de difficultés et seulement après une période de temps considérable, et non sans la présence d'une bonne part d'erreur ${ }^{31}$.

Mais il y a encore plus, car même les préceptes relatifs au prochain (seconde table) ne peuvent pas toujours être déduits très facilement. Et pour expliquer la convenance de la révélation portant sur ces préceptes, saint Thomas explique:

En ce qui touche les préceptes moraux, la raison humaine ne pouvait se tromper sur les préceptes les plus généraux de la loi naturelle, dans leur teneur universelle, encore que l'accoutumance du péché troublât son regard dans le détail de l'action. Sur les autres préceptes moraux qui dérivent à la manière de conclusions des principes généraux de la loi naturelle, beaucoup d'esprits tombaient dans l'erreur au point de considérer comme licite ce qui est réellement mauvais. Contre ces deux sortes de défaillances l'homme devait être secouru par la garantie de la loi divine ${ }^{32}$.

On aura sans doute reconnu, dans le texte qui précède, la doctrine que saint Thomas applique également à la question de savoir si les préceptes de la loi naturelle sont immuables et s'ils peuvent être effacés du coeur de l'homme. La partie de sa réponse qui porte sur les préceptes secondaires s'applique donc au Décalogue:

Quant aux préceptes secondaires, la loi naturelle peut être effacée du coeur des hommes soit en raison de propagandes perverses, de la même façon dont les erreurs se glissent dans les sciences spéculatives au sujet de conclusions nécessaires; soit comme conséquences de coutumes dépravées et d'habitudes de corruption morale. C'est ainsi que certains individus, au témoignage de saint Paul, ne considéraient pas le brigandage ni les vices contre nature comme des péchés ${ }^{33}$.

Si donc la dérivation des préceptes secondaires ne requiert qu'une «brève réflexion», on ne saurait pourtant dire que cette réflexion soit simple et facile. Ajoutons encore les quelques observations suivantes. Premièrement, en ce qui a trait à la première table du Décalogue, saint Thomas fait remarquer qu'un

31. S.C.G., 1. I, c.4.

32. Q. 99, a.2, ad 2 .

33. Q. 94, a.6. Un exemple semblable est aussi utilisé à la q. 94 , a.4, où saint Thomas précise si la vérité pratique peut être la même pour tout le monde en matière de principes pratiques et de conclusions plus ou moins rapprochées. 
élément cérémoniel s'ajoute parfois au précepte moral proprement dit, par exemple la détermination d'un jour spécial consacré au culte dû à Dieu ${ }^{34}$. Un précepte de ce genre ne peut être atteint simplement par mode de conclusion; le raisonnement procède ici plutôt par mode de détermination, et il en sera de même, semble$t-i l$, pour tous les préceptes ajoutés au Décalogue ${ }^{35}$. Deuxièmement, en ce qui concerne les omissions apparentes aux préceptes du Décalogue, par exemple l'amour de soi-même ou encore celui que les parents doivent à leurs enfants, saint Thomas suggère trois réponses qui paraissent partiellement incompatibles et parmi lesquelles il faudrait choisir. Ainsi, le Décalogue ne contient aucun précepte portant sur l'amour de soi-même:

\begin{abstract}
parce que sur ce point, la loi naturelle gardait toute sa force; - ou encore parce que l'amour de soi est inclus dans l'amour de Dieu et du prochain, vu que s'aimer vraiment c'est se rapporter à Dieu. (...)
\end{abstract}

Mais que l'homme soit tenu d'une dette rigoureuse envers soimême et non envers autrui, cela ne saute pas aux yeux . . . Aussi les préceptes qui interdisent à l'homme de se manquer à soi-même sont-ils communiqués au peuple par l'intermédiaire des sages ${ }^{36}$.

Dans la première hypothèse, on aurait affaire à un précepte commun accessible à la seule raison naturelle; dans la seconde hypothèse, il s'agirait encore d'un précepte commun inclus dans un précepte antérieur qui implique à son tour la Révélation ou la foi; dans la troisième hypothèse, il s'agirait cette fois d'un précepte tertiaire ou d'une conclusion éloignée.

Troisièmement, il faut encore faire remarquer que, même si les préceptes du Décalogue constituent des conclusions rapprochées des préceptes premiers et ne nécessitent qu'une brève réflexion, on y décèle néanmoins un certain ordre d'importance et de plus ou moins grande nécessité ou évidence. Car, explique saint Thomas:

Il paraît bien qu'une donnée soit d'autant plus accessible à la raison que son contraire est davantage et plus gravement opposé à la raison. Et comme c'est dans la fin que l'ordre de la raison trouve son

36. Q. 100, a. 5 , ad 1 . 
principe, ce qui par-dessus tout s'oppose à la raison, c'est le dérèglement au regard de la fin ${ }^{37}$.

C'est donc sur la base d'une application diversifiée de ce principe que s'expliquent l'ordre réciproque et l'ordre interne des deux tables du Décalogue. Le Père J. Tonneau résume et commente avec justesse:

Les devoirs envers Dieu avant les devoirs envers le prochain et parmi ceux-ci priorité des devoirs envers les parents, les autres se suivant par ordre de gravité décroissante, sans que s'impose comme principe d'ordre la différence des préceptes affirmatifs et des préceptes négatifs ${ }^{38}$.

Quatrièmement, la distinction susmentionnée entre préceptes affirmatifs et préceptes négatifs ne semble pas avoir une portée considérable sur notre propos, même s'il est vrai que les préceptes négatifs peuvent paraître plus évidents à cause de leur portée plus universelle $e^{39}$. Et il faudrait, semble-t-il, en dire autant de la question des dispenses apparentes aux préceptes du Décalogue $e^{40}$.

Il reste cependant à considérer, comme reliées au Décalogue, quatre questions plus complexes que nous allons considérer brièvement et séparément: le droit de propriété, la régulation du mariage, le droit des gens, la loi civile en général.

5 - Le droit de propriété. Chacun se souvient que le $7 \mathrm{e}$ commandement interdit le vol, tandis que le 10 e interdit même de convoiter le bien du prochain. Or, demande justement le Père M.-J. Laversin: «Comment le vol serait-il interdit par la loi naturelle si la propriété ne relevait pas, elle-même, de cette loi? ...41» À première vue, il semblerait donc que le droit de propriété relève des préceptes premiers du droit naturel. Mais qu'en est-il en réalité? Si l'on se réfere aux textes où saint Thomas en traite expressément, on constatera, avec J. Maritain, que

la question de la propriété comporte trois moments successifs: dans le premier moment, saint Thomas montre que l'homme considéré

37. Q. 100, a.6.

38. S. Thomas d'Aquin, La loi ancienne, tome I, Desclée, Paris, 1971, p. 188.

39. Q. 100 , a. 4 ; a. 6 , ad 2; a. 7 , ad 1 : «L'obligation de ne pas faire de tort s'étend à plus de personnes que celle de rendre service.»

40. Q. 94, a.5, ad 2; q. 97, a.4, ad 3; q. 100, a.8. Les trois exemples cités se rapportent à des commandements relatifs au prochain.

41. S. Thomas d'Aquin, La loi, Desclée, Paris, 1935, p. 251. 
dans son type spécifique a un droit général d'approptiation à l'égard de tous les biens matériels...

Au second moment, saint Thomas montre que l'appropriation des biens extérieurs doit normalement avoir lieu sous forme personnelle...

Il reste cependant que l'usage (usus) de ces biens doit être commun...

Le troisième moment a rapport aux modalités particulières de la propriété $^{42}$.

Mais les deux premiers «moments» comportent chacun deux étapes. Voyons le premier moment. Dans une première étape, saint Thomas montre d'abord qu'une chose peut être dite de droit naturel

d'une part, parce que la nature l'y incline, par exemple: "Il ne faut pas être injuste envers autrui.» D'autre part, parce que la nature ne suggère pas le contraire . . C'est précisément de la sorte que «la possession commune de tous les biens et la liberté identique pour tous» sont dites de droit naturel: en ce sens que la distinction des possessions et la dépendance ne sont pas imposées par la nature, mais par la raison des hommes pour l'utilité de la vie humaine. En cela la loi de nature n'est point modifiée sinon par mode de complément ${ }^{43}$.

Dans une deuxième étape, saint Thomas explique que, quant à leur nature, les choses extérieures ne sont pas soumises au pouvoir de l'homme, mais de Dieu seul; mais que, quant à leur usage, l'espèce humaine possède un pouvoir de gérance sur les choses de la nature, puisque les êtres imparfaits existent pour les plus parfaits ${ }^{44}$.

Au second moment, saint Thomas va parler de ce complément au droit naturel qui justifie la distinction des possessions ou l'appropriation personnelle. Ici encore l'argumentation peut être développée en deux étapes. Dans la première, saint Thomas part du principe que le droit, ou «juste» naturel,

c'est ce qui par nature s'ajuste ou se proportionne à autrui. Mais cela peut arriver de deux manières: soit qu'on envisage la chose absolument et en soi, par exemple un mari qui, comme tel,

\footnotetext{
42. J. Maritain, Du régime temporel et de la liberté, Desclée, Paris, 1933, p. 230-232. Voir IIaII ae, q. 66 , a. 1 et a. 2 .

43. Q. 94, a.5, ad 3.

44. $I^{a} I I^{a}$, q. 66, a. 1 .
} 
s'adapte à une femme pour en avoir des enfants, ou un père à son fils pour le nourrir; soit qu'on l'envisage non plus absolument, mais relativement à ses conséquences, par exemple la propriété privée ${ }^{45}$.

Résumons la suite du texte: un champ considéré absolument ne demande pas à appartenir à un tel plutôt qu'à tel autre; mais, au point de vue de sa culture et de son usage dans la paix, il vaut mieux qu'il appartienne à un individu déterminé. Et saint Thomas d'ajouter: le droit naturel qui découle de la considération des choses prises absolument est celui que nous partageons en commun avec les animaux; mais celui qui découle de la considération des conséquences, laquelle considération n'est propre qu'à l'homme à cause de sa raison, dérive de ce qui est spécifique à l'homme et s'appelle le «droit des gens". Comme on le voit déjà, ce texte a une portée sur les autres choses dont on parlera plus loin.

À la seconde étape, saint Thomas va préciser à la fois ce qui justifie la propriété privée et jusqu'où elle s'étend. Il s'agit, comme on l'a vu plus haut, du pouvoir de gérer des biens possédés en propre et d'en disposer. La propriété privée lui apparaît nécessaire pour trois raisons d'ordre social: la nature des soins qu'il faut apporter à la gestion des biens, l'élimination de la confusion dans le travail, la réalisation de la paix publique ${ }^{46}$. Mais la nature même de ces trois raisons d'ordre social fait que l'usage ultime de la propriété privée doit rester subordonné aux fins de la vie en société et à un certain usage commun.

Il ne restera enfin, au troisième moment, qu'à déterminer les modalités concrètes de l'exercice du droit de propriété. Cet aspect de la question relève plutôt du droit positif ${ }^{47}$, alors que la détermination du caractère privé de la propriété se rattache au droit naturel dérivé, dit droit des gens, et que le droit général d'appropriation fait partie du droit naturel premier. Il semble alors que rien ne s'opposerait à ce que l'interdiction du vol (précepte secondaire) se rattache à la fois au droit naturel premier et à un droit des gens promulgué sous forme de coutume.

6 - Les formes du mariage. La question du mariage présente des problèmes peut-être encore plus difficiles. Mais un premier

45. $I^{a} I^{a e}, \mathrm{q} .57$, a. 3 .

46. $I^{a} l I^{a e}$, q. 66, a. 2 .

47. Id., ad 1 . 
point peut être tranché assez rapidement. Comme on l'a vu plus haut, le fait même de l'union des deux sexes, indépendamment de toute modalité, relève du droit naturel premier, c'est-à-dire des inclinations naturelles et de la nature des choses considérées absolument ou en elles-mêmes ${ }^{48}$.

Mais en ce qui concerne la forme même du mariage et de l'interprétation plus précise des inclinations et nécessités naturelles, il n'en va plus aussi facilement. En effet, l'interdiction de commettre l'adultère ou de convoiter la femme du prochain ne préjuge en rien du nombre d'épouses ou du nombre de maris qu'il peut être légitime d'avoir. On connaît toutefois la théorie habituellement présentée comme la doctrine thomiste et tirée de premiers écrits de saint Thomas. Elle consiste à attribuer deux fins au mariage. La fin première, à savoir la procréation et l'éducation des enfants, serait tirée du genre et rattachée aux préceptes primaires du droit naturel. C'est à cette fin que s'opposerait surtout la polyandrie. La fin secondaire, à savoir le secours mutuel, serait tirée de l'élément spécifique de l'homme et rattachée aux préceptes secondaires du droit naturel. C'est à cette fin que s'opposerait la polygamie ${ }^{49}$, et que se rattacherait l'exigence d'indissolubilité.

Cette théorie ne manque sans doute ni d'attrait ni de valeur. Elle soulève cependant deux ordres de difficultés. D'une part, quant à sa fidélité à la pensée de saint Thomas d'Aquin. En effet, les études les plus récentes sur les textes de saint Thomas montrent de manière décisive que la célèbre distinction entre fin primaire et fin secondaire du mariage a été définitivement abandonnée par lui dès la rédaction de la Somme contre les Gentils, et qu'elle ne se trouve nulle part dans les textes ultérieurs de la Somme théologique. La distinction entre préceptes primaires et préceptes secondaires du droit naturel demeure, mais elle est désormais fondée sur le critère plus subtil du degré d'évidence des divers préceptes ${ }^{50}$. D'autre part, il faut considérer la cohérence interne

48. IIII $^{a t}$, q. 57, a. 3 .

49. Suppl., q. 41, a. 1; q. 65, a.1. J.M. Aubert, Le droit romain dans l'oeuvre de Saint Thomas, Paris, J. Vrin, 1955, p. 109-113.

50. R.A. Armstrong, Primary and Secondary Precepts in Thomistic Natural Law Teaching, Martinus Nijhoff, The Hague, 1966, p. 82-83. M. B. Crowe, The Changing Profile of the Natural Law, Martinus Nijhoff, The Hague, 1977, p. 182-183. 
de la pensée ultérieure de saint Thomas. Car, ainsi que le fait remarquer M.B. Crowe:

The division of man's natural inclinations into three groups cuts across the division, found in the Commentary on the Sentences, between primary and secondary precepts of the natural law. There are primary and secondary precepts belonging to each of the three grades of inclination ${ }^{51}$.

Il peut donc se faire qu'un même précepte soit primaire dans son propre ordre d'inclination, mais secondaire par rapport à un autre ordre. En conséquence, ne faudrait-il pas revoir avec plus de précision la distinction des préceptes primaires et secondaires du droit naturel, et l'application qui peut en être faite à des problèmes tels que l'unité et l'indissolubilité du mariage? Qu'advient-il, par exemple, si on révise en conséquence la théorie du mariage en considérant le secours mutuel et l'amitié des époux non plus comme une fin secondaire, mais comme une fin vraiment première dans son ordre? À considérer les personnes absolument et en elles-mêmes, le mariage ne doit-il pas être une communauté d'égaux?

Le prochain n'est pas la fin de l'homme, et il n'y a pas lieu pour celui-ci de se régler intérieurement en fonction du prochain. Ce serait une attitude servile ${ }^{52}$.

Que l'homme puisse, quand même, abandonner à son gré la femme qu'il a prise, le mariage ne serait plus une société d'égaux, mais le despotisme de l'homme et l'esclavage de la femme ${ }^{53}$.

Mais les autres questions relatives au mariage sont peut-être susceptibles de recevoir certains éclaircissements de l'examen rapide de nos deux derniers sujets de discussion.

7 - Le droit des gens. Précisons immédiatement que, pour saint Thomas d'Aquin, il ne s'agit pas du droit international, mais d'un droit en quelque sorte intermédiaire entre le droit naturel au sens restreint et le droit positif ou civil. C'est un droit naturel en ce sens qu'il dérive également des préceptes premiers par mode de conclusions rapprochées; mais il se distingue du droit naturel restreint, que l'homme est dit posséder en commun

51. M.B. Crowe, op. cit., p. 179. Dans le même sens, voir B.H. Merkelbach, Summa Theologiae Moralis, Paris, Desclée de Brouwer, 3e éd., 1938, tome I, p. 234, n. 1.

52. Q. 104, a.1, ad 3.

53. S.C.G., I. II, ch. 123. 
avec les animaux, en ce qu'il est dérivé de l'élément spécifique de l'homme et ne s'applique qu'aux rapports des hommes entre eux. Il s'en distingue encore en ceci que le droit naturel restreint considère les choses absolument et en elles-mêmes, de même que celles auxquelles la nature incline, tandis que le droit des gens considère les choses quant à leurs conséquences et à ce qui peut être le plus indispensable et le plus utile pour le bien-vivre. Bref, c'est le droit qui est observé par tous les peuples et entre tous les hommes, sans pourtant requérir d'institution spéciale; il ne doit son existence qu'à la seule raison naturelle. Parmi les coutumes sociales qui en relèvent, saint Thomas mentionne l'existence de l'autorité politique, l'esclavage, le droit de propriété, le respect des contrats ou traités, la recherche de la justice dans les achats et les ventes ${ }^{54}$. Et il faudrait peut-être y ajouter tous les préceptes de la deuxième table du Décalogue, c'est-à-dire les préceptes relatifs au prochain.

L'exemple le plus instructif et le plus clair parmi tous ceux qui sont mentionnés ci-dessus est probablement celui de la propriété privée. Il fait ressortir trois choses: d'abord, les différents niveaux de réflexion qui doivent accompagner les différentes sortes de droit: le droit naturel restreint, le droit des gens et le droit positif; ensuite, l'évolution historique qui peut se produire à l'intérieur de chacune de ces sortes de droit; la nécessité, enfin, de tenir compte des circonstances contingentes au moyen des déterminations concrètes de la loi positive ${ }^{55}$.

Mais il ne facilite guère la question d'avoir à distinguer de plus, à l'intérieur du droit des gens, ce qui découle de la nature humaine permanente et ce qui découle de la nature déchue. Dans le premier cas, au dire de saint Thomas, on obtient l'autorité politique de même que certaines inégalités naturelles; dans le second cas, on obtient le fait de l'esclavage, qui relève également du droit positif par son caractère de peine et par ses modalités. En

54. Q. 95, a. 4 et ad. 1; q. 94, a. 3; q. 94, a. 5, ad. 3; Ilallae, q. 12, a.2; q. 57, a.3. J. Maritain accentue peut-être un peu trop la différence entre l'aspect intuitif du droit naturel restreint et l'aspect déductif du droit des gens (L'Homme et l'État, p. 91). Contre cette opinion, voir $\mathrm{P}$. Sigmund, Thomistic Natural Law and Social Theory, dans Calgary Aquinas Studies, Ed. by A. Parel, Pontifical Institute of Mediaeval Studies, Toronto, 1978, p. 69. In Etbi., V, 1. 12, n. 1019: Pacta sunt servanda.

55. Yves R. Simon, The Tradition of Natural Law, Fordham University Press, New York, p. 154. 
outre, même si la portée de l'esclavage est restreinte à certaines choses relatives au corps, l'esclave ne peut néanmoins faire partie du corps politique ${ }^{56}$. Voilà sans aucun doute une série d'affirmations qui, si elle relève du droit naturel secondaire, appelle aussi quantité de nuances qui lui enlèvent tout caractère de réflexion simple.

C'est au niveau du droit des gens, semble-t-il, que doit se situer l'évaluation du degré de nécessité des diverses inclinations humaines d'ordre familial ou social. Par exemple, comment juger du droit des peuples à l'autodétermination? On ne peut, ici, que soulever la question ${ }^{57}$. Mais il est certain que cette évaluation ne peut être faite sans la considération de ce qui est nécessaire ou utile au bien commun humain.

8 - Le droit positif. Ainsi qu'on vient de le voir, le droit naturel au sens strict et le droit des gens se transforment presque insensiblement en droit positif soit par la coutume, soit par la législation proprement dite. Il ne saurait en être autrement, puisque toute action doit être posée dans des circonstances concrètes toujours particulières. C'est pourquoi le droit positif prend nécessairement, pour saint Thomas, la forme d'une détermination des préceptes plus généraux du droit naturel strict et du droit des gens.

De même donc que la détermination du précepte général de rendre un culte à Dieu est assurée par les préceptes cérémoniels, de même la détermination du précepte général d'observer la justice parmi les hommes est assurée par les préceptes judiciaires ${ }^{58}$.

Ce droit comporte donc très souvent des éléments qui sont eux-mêmes tirés du droit naturel et du droit des gens. Cependant, considéré en lui-même, c'est un droit propre à chaque entité politique non seulement dans le détail de sa législation, mais d'abord en ce qui concerne le choix fondamental de sa propre constitution ou forme de gouvernement. C'est un droit qui doit essentiellement tenir compte de sa propre adaptation aux coutu-

\footnotetext{
56. I I ${ }^{a}$., q. 96, a.4; $I^{a} I I^{a e}$, q. 98, a.6. ad 2;II $I^{a}{ }^{a e}$, q. 104, a.5; Suppl., q. 52, a. 1, ad 3; De Reg. Pr., I, c. 1 .

57. Sans doute, l'exercice d'un tel droit n'est pas obligatoire; mais considérée en elle-même, en quoi la diversité des peuples serait-elle un moindre bien pour l'humanité que la diversité des individus?

58. Q. 99, a.4.
} 
mes, temps et lieux qui conditionnent son application, et qui est redevable pour beaucoup à l'apport de l'expérience et à l'avis des experts en la matière. Dans le prolongement du droit naturel, il s'étend à un grand nombre de choses d'abord indifférentes en elles-mêmes, mais qui cessent de l'être une fois qu'il a été statué à leur sujet ${ }^{59}$.

Relèvent donc plus précisément du droit positif toutes les déterminations qui fixent les modalités concrètes des actions demandant à être réglées par la justice. Saint Thomas en donne les quelques exemples suivants: le voleur doit recevoir telle peine; il faut fixer telle rançon pour les captifs; il faut rendre tel hommage à un bienfaiteur. Chacun pourrait allonger la liste à son gré, et aura compris qu'il s'agit ici d'un droit essentiellement modifiable qui doit être adapté aux changements survenant dans la condition des hommes ${ }^{60}$. Son domaine s'étend à toutes les branches de notre droit moderne.

Quant aux modalités de l'organisation sociale et politique élaborée dans le sillon du droit naturel, saint Thomas prend son point de départ dans une conception hiérarchique de l'univers qui doit beaucoup à ses prémisses théologiques et qui se répercute tant dans l'ordre social que dans l'ordre politique. Qu'il suffise ici de dire qu'il croit en la nécessité, pour mener l'homme à sa fin, de deux sociétés distinctes dont l'une, l'Église, découle de la loi divine, et l'autre, la société politique, dérive de la loi naturelle. Dans l'ordre social, le cadre lui en est fourni par les quatre types de rapports déjà identifés par les préceptes judiciaires de l'ancienne Loi. Ce sont les rapports entre chefs et sujets; les rapports de sujets à sujets; les rapports entre citoyens et étrangers; et enfin, les rapports domestiques. Voilà le cadre à l'intérieur duquel se situe l'expression de sa préférence pour la monarchie tempérée et une forme de gouvernement mixte. Mais on voit facilement jusqu'à quel point ces précisions peuvent se situer au-delà des purs préceptes du droit naturel ${ }^{61}$.

59. Q. 95, a. 2; q. 3; a.4; q. 99, a.5; $I^{a} I I^{a e}$, q. 10 , a. 10; q. 12 , a.2; In Eth., I. V, leç. 12 , nn. $1020-1022$.

60. Q. 95, a.2; q. 97 , a. 1; In Eth., 1. V, leç. 12, nn. 1023-1024.

61. $I^{a} P$., q. 108 , a. $2 ; I^{2} I I^{*}$, q. 72 , a.4; q.95, a.4; q. 104 , a.4; q. 105 , a.1. K. Archibald, "The Concept of Social Hierarchy in the Writings of St. Thomas Aquinas", dans The Historian, 1949 , vol. 12 , no. 1 , p. $28-54$. 
Les tenants du volontarisme et du positivisme juridique ont bien vu la part d'arbitraire que comporte la loi et le rôle qu'y joue la volonté du législateur; ils ont également saisi que la large part de rationalisation qu'elle incorpore permet d'en faire une étude scientifique ${ }^{62}$. Mais ils semblent avoir oublié, d'une part, le lien inévitable que le droit positif conserve en dépit de lui-même avec le droit naturel, et, d'autre part, le fait que le droit positif n'est pas seulement une science mais bien davantage un art. Il s'agit, en effet, d'énoncer moins des lois sur l'homme que des lois pour l'homme. Ce que saint Thomas disait déjà de la science morale trouverait ici, à plus forte raison, une nouvelle application:

Si l'étude de cette science se bornait à la seule connaissance de la vérité, elle serait de peu d'utilité ${ }^{63}$.

Si l'on veut amener quelqu'un à observer des préceptes, on doit mettre en avant les motifs auxquels il est sensible ${ }^{64}$.

Il est manifestement vain de chercher à résumer en conclusion toutes les nuances essentielles des analyses qui précèdent. Qu'il suffise donc de faire ressortir les points suivants. En premier lieu, c'est à partir des grands genres d'inclinations naturelles que, pour saint Thomas, la raison humaine élabore les premiers préceptes du droit naturel. Le P. Sertillanges a donc raison d'écrire:

Les préceptes premiers sont relatifs aux fins générales, et à l'essentiel de la fin principale en chaque genre de pratique. Les préceptes seconds concernent les fins secondaires ou l'accessoire des fins principales ${ }^{65}$.

En second lieu, il faut noter qu'en ce qui concerne les préceptes secondaires (v.g. le Décalogue), dérivés par mode de conclusion, il faut se garder d'interpréter la déduction comme un processus purement abstrait et pouvant se passer du recours à l'expérience pour la découverte des concepts particuliers entrant dans l'énoncé des préceptes secondaires ${ }^{66}$.

En troisième lieu, on aura encore remarqué, au sujet des préceptes secondaires dérivés par mode de conclusion rapprochée

62. H. Rommen, Le Droit naturel, Histoire-Doctrine, Paris, Egloff, 1945, 310 p.

63. In Eth., 1, II, leç. $2, \mathrm{n}^{\circ} 256$.

64. Q. 99, a.6.

65. R.P. Sertillanges, La philosophie morale de St Thomas d'Aquin, Paris, Aubier, 1961, p. 105.

66. F.C. Copleston, Aquinas, Penguin Books, 1955, p. 223-224. 
des préceptes primaires, qu'il existe un certain recoupement entre les préceptes du droit naturel restreint et les préceptes du droit des gens. Peut-être n'est-il pas téméraire d'émettre la suggestion suivante. Il semble qu'en ce qui concerne les préceptes du premier groupe, la considération de conditions particulières n'entre pas en ligne de compte dans leur formulation même, mais doive entrer en ligne de compte pour ce qui est de leur application concrète; tandis que pour les préceptes du droit des gens, la considération de conditions contingentes en elles-mêmes mais générales de fait, doive entrer en ligne de compte dans la formulation même des préceptes $^{67}$.

En dernier lieu, à mesure que la réflexion de l'intelligence se porte moins sur les éléments essentiels d'une action, mais davantage sur ses conséquences moins immédiates et sur les conditions contingentes ou purement indifférentes qui l'accompagnent, plus on pénètre à l'intérieur du processus de dérivation par mode de détermination et plus on rejoint par là les différents domaines du droit positif. Voilà pourquoi il nous apparaît que la théorie du droit naturel et du droit positif chez saint Thomas d'Aquin ne peut en aucune façon être décrite comme une théorie purement déductive, mais qu'au contraire, la part du raisonnement empirique et du mode de détermination y occupe de loin la place prépondérante.

Département de philosophie

Université d'Ottawa

67. J. Maritain, Les droits de l'bomme et la loi naturelle, dans Oeutres (1940)1963), Choix et notes par Henry Bars, Desclée de Brouwer, 1978, p. 203. 